\title{
Dorsal Anterior Cingulate Cortical Thickness Is Associated with Improvement in Worry Severity Following Treatment in Late-Life Generalized Anxiety Disorder
}

Author(s): Anusha Rangarajan ${ }^{\mathrm{a}}$, Helmet T. Karim ${ }^{\mathrm{b}}$, Dana L. Tudorascu ${ }^{\mathrm{c}}$, Sarah Walker ${ }^{\mathrm{b}}$, Howard Aizenstein ${ }^{\mathrm{a}, \mathrm{b}}, *$ Carmen Andreescu ${ }^{\mathrm{b}}$

\author{
Affiliation(s): \\ aDepartment of Bioengineering, University of Pittsburgh, Pittsburgh, PA, USA \\ ${ }^{b}$ Department of Psychiatry, University of Pittsburgh, Pittsburgh, PA, USA; 'Department \\ of Medicine, Graduate School of Public Health, Pittsburgh, PA, USA \\ *Corresponding author: Carmen Andreescu, MD; University of Pittsburgh, Department \\ of Psychiatry, Western Psychiatric Institute and Clinic, 3811 O'Hara Street, phone 412 \\ 246 5698, fax 412586 9111, e-mail: Andrcx@upmc.edu
}




\begin{abstract}
We recently showed that late-life participants with generalized anxiety disorder (GAD), compared with non-anxious elderly, had lower cortical thickness in the dorsal anterior cingulate cortex (ACC) and the orbitofrontal cortex (OFC). In this follow-up study, we investigated the association between cortical thickness and clinical markers of treatment response in a subgroup from that same sample. Twelve GAD participants were randomly selected and were included in an open label twelve-week treatment with citalopram and pre-/post-treatment magnetic resonance imaging (MRI). Cortical thickness measurements were obtained with Freesurfer. Six regions were selected based on previous results: left dorsal ACC, left rostral ACC, left and right medial orbitofrontal, right pars triangularis and right pars opercularis. A two-tailed paired t-test (non-parametric Wilcoxon Signed Rank test) was used to compare pre- and post-treatment for all clinical variables. For each region of interest (ROI), the Pearson correlation coefficient was estimated between pretreatment cortical thickness and change in Penn State Worry Questionnaire (PSWQ) from pre- to post-treatment. Our results indicate that greater left pre-treatment dorsal ACC cortical thickness was associated with improvement in worry severity post-treatment. In late-life $\mathrm{GAD}$, greater treatment response is associated with greater cortical thickness in areas pivotal for emotion regulation.
\end{abstract}

Keywords: Generalized anxiety disorder, biomarkers of treatment response, structural changes in GAD

\title{
Key points:
}

- We associated cortical thickness with clinical markers of treatment response in late-life Generalized Anxiety Disorder (GAD) participants

- Worry improvement post-treatment was associated with greater dorsal Anterior Cingulate Cortex (dACC) cortical thickness

- Treatment response was associated with cortical thickness of areas pivotal for emotion regulation 


\section{Introduction}

Generalized anxiety disorder (GAD) is one of the most common anxiety disorders in older adults with a prevalence rate of 3.1 [9] - 11\% [43]. Late-life GAD is characterized by excessive and difficult to control worry, as well as irritability, difficulty concentrating, sleep disturbance, fatigue and muscle tension [24]. Late-life GAD has been associated with impaired cognitive function, increased depression risk and decreased quality of life $[4,8,37,41]$. While most anxiety disorders' prevalence decreases in late-life [7], GAD has a second peak of incidence after age 50 [27], a finding which may be associated to age-related brain structural changes.

Only a few studies have explored the structural neuroanatomical changes associated with late-life GAD. One study reported greater orbitofrontal cortex volume and greater worry severity in older adults with GAD and non-anxious controls [33]. Previously in a whole brain analysis we explored the differences between gray matter indices (cortical thickness and volume) in late-life GAD and non-anxious elderly participants. GAD participants had lower cortical thickness in the orbitofrontal cortex (OFC) and dorsal anterior cingulate cortex, and lower cortical volume in inferior frontal gyrus (ACC) [2]. Higher worry severity was associated with lower gray matter thickness in BA24 region (left dorsal and rostral ACC). After controlling for age, only the association between worry severity and cortical thickness of rostral ACC remained significant. This study suggests that late-life GAD is associated with gray matter in areas involved in emotion regulation.

These findings are relevant in the context of the emotion dysregulation theory of GAD [30]. This theory suggests that GAD is associated with both deficits in emotion generation (including a tendency for strong emotional responses mediated by increased perceived threats) and in emotion regulation (rigid attention responses to emotional stimuli and poor compensatory strategies) [10, 30].

Deficits in emotion regulation in anxiety disorders have been associated with structural prefrontal changes [14, 39], but it is less known if these structural changes interfere with treatment response. Thus, we tested the association between cortical thickness in regions previously identified and changes in clinical markers of treatment response in late-life GAD. For this purpose, we analyzed pre- and post-treatment 
structural indices in twelve older GAD participants who received open-label treatment with citalopram for twelve weeks. We hypothesized that greater cortical thickness in the dACC, OFC and PFC would correlate with treatment response as measured by changes in worry severity.

\section{Materials and Methods}

\subsection{Study Design and Participants}

Participants were recruited from an NIMH-funded trial ("Structural and functional neuroanatomy of late-life GAD"). This study was approved by the University of Pittsburgh Institutional Review Board. Twelve GAD participants were randomly selected from this larger sample and were included in an open-label twelve-week treatment with

citalopram and pre-/post-treatment magnetic resonance imaging (MRI). Older adult GAD participants (age 60 and over) had a principal diagnosis of GAD for at least six months according to the Structured Clinical Interview for DSMIV (SCID) [17] and a score of 17 or higher on the Hamilton Anxiety Rating Scale (HARS) [22] at the time of their pretreatment MRI scan. Participants were allowed to receive non-psychotropic medications.

\subsection{Assessments and treatments}

Participants were additionally evaluated with the Penn State Worry Questionnaire (PSWQ) [31], the rumination subscale from the Response Style Questionnaire (RSQ) [34], the Five Factor Inventory Neuroticism scale [12] and the Hamilton Depression Rating Scale (HDRS) [23]. We included a rumination subscale as worry and rumination are distinct factors in joint factor analyses [25], but they share a common higher order factor in the form of perseverative thought [36]. Global medical burden was assessed using the Cumulative Illness Rating Scale for Geriatrics (CIRS-G) [32]. Participants also had comprehensive neuropsychological evaluation using the Repeatable Battery for the Assessment of Neuropsychological Status (RBANS) [15] and Mini Mental Examination Scale (MMSE)[20]. The total duration of illness was determined based on participants' self-report. All raters - for both clinical and neuropsychological measures - were masterlevel clinicians. Interrater reliability, as measured by intraclass correlation coefficient [24], was high: $\mathrm{MMSE}=0.98, \mathrm{HARS}=0.97$, and $\mathrm{HDRS}=0.93$. 
Participants were psychotropic free (about 2-6 weeks depending on their medication) at the pre-treatment scan but were allowed non-psychotropic medications. GAD participants received treatment with citalopram - mean final dose (Mean (SD) $=$ 19.17mg (5.15)). Remission was defined as a HARS score of 7 or below. The University of Pittsburgh Institutional Review Board approved the study and all participants provided written informed consent.

\subsection{Exclusion criteria}

Patients with other anxiety disorders were included if GAD was the principal diagnosis (based on severity and duration), as were patients with a past history of alcohol or substance abuse that was in full remission for at least 3 months. Although lifetime comorbid unipolar depression was allowed if GAD was the primary diagnosis (based on duration), none of the participants included in this study had current Major Depressive Disorder at the time of scanning. Participants with MMSE scores of 24 or lower, or with a clinical diagnosis of dementia were excluded. Other exclusion criteria were lifetime psychosis or bipolar disorder, increased suicide risk (e.g. current ideation), a history of stroke or other severe neurologic disease, medical instability according to review of medical chart data, ongoing psychotherapy, and current antidepressant or anxiolytic use.

\subsection{MRI Data Acquisition}

Imaging data were collected pre- and post-treatment at the University of Pittsburgh Magnetic Resonance Research Center using a 3-Tesla Siemens Trio TIM scanner with a 32-channel head coil. High-resolution anatomical images (T1-weighted magnetizationprepared rapid gradient echo MPRAGE) were collected over four minutes and 43 seconds using the following parameters: FOV $=256 \times 254 \mathrm{~mm}$, voxel size $1 \mathrm{~mm}^{3}$ (isotropic), $\mathrm{TI}=900 \mathrm{~ms}, \mathrm{TR} / \mathrm{TE}=2000 / 3.43 \mathrm{~ms}$, flip angle $=9^{\circ}$.

\subsection{Freesurfer segmentation}

Freesurfer (v 6.0) [13] [18] was used for cortical thickness segmentation. In the surface based segmentation stream, the T1 weighted image was registered to the MNI305 template [11] and was bias corrected. After white matter was classified, the intensity 
gradients between white and gray matter generated the surface. The pial surface was inflated and registered to a spherical atlas based on gyral folds to obtain the cortical labeling.

\subsection{Cortical Thickness computation}

Cortical thickness measurements were obtained from Freesurfer segmentations. Six regions were selected based on a previous study [2]: left dorsal and rostral ACC, left and right medial orbitofrontal, right pars triangularis and right pars opercularis. We normalized the cortical thickness measurements from each region using the average cortical thickness of the brain.

\subsection{Statistical Analysis}

A two-tailed paired t-test (non-parametric Wilcoxon Signed Rank test) was used to identify differences between pre- and post-treatment for all clinical variables (PSWQ, HAM-A, HAM-D, MMSE, CIRS-G, RSQ, and RBANS). For each ROI, we tested whether there was a significant difference between pre- and post-treatment normalized cortical thickness. For each ROI, the Pearson correlation coefficient was estimated between pre-treatment cortical thickness and change in PSWQ from pre- to posttreatment. We accounted for other clinical variables (RSQ, HAMD, HAMA, comorbid depression lifetime duration, total duration of GAD) as covariates if the Pearson correlation with baseline cortical thickness was significant. The Benjamini-Hochberg procedure was used (for multiple comparisons correction) to control the false-discovery rate at an $\alpha<0.05[5]$.

\section{Results}

The participants had a mean age of 66 and were all Caucasian (baseline mean HDRS=21, PSWQ=58). All participants responded to treatment. Due to small sample sizes, we have tested for pre-/post-treatment differences using Wilcoxon Signed Rank test (Table 1). Except for RSQ, all the other clinical variables were significantly lower post-treatment [HAMA ( $Z=-3.07, p=0.002), \operatorname{HAMD}(\mathrm{Z}=-3.08, \mathrm{p}=0.002)$, PSWQ $(\mathrm{Z}=2.59, \mathrm{p}=0.01)$, and 
RSQ $(Z=-1.49, p=0.14)]$. The difference between the treated sample in this analysis $(\mathrm{N}=12)$ to the rest of the sample $(\mathrm{N}=8)$ is shown in Table 2.

\begin{tabular}{|l|c|c|c|c|}
\hline Demographics and Clinical data & $\begin{array}{c}\text { N=12 } \\
\text { Pre-treatment }\end{array}$ & $\begin{array}{c}\text { N=12 } \\
\text { Post-treatment }\end{array}$ & $\mathbf{Z}$ & p-value \\
\hline Age [Mean (SD)] & $66.33(5.61)$ & - & - & - \\
\hline Gender (F) & 10 & - & - & - \\
\hline Race & $12(\mathrm{~W})$ & - & - & - \\
\hline Education [Mean (SD)] & $14.75(2.59)$ & - & - & - \\
\hline HAM-A [Mean (SD)] & $21.42(4.42)$ & $6.16(4.39)$ & $\mathrm{Z}=-3.07$ & $p=0.002$ \\
\hline HAM-D [Mean (SD)] & $13.4(3.49)$ & $4.33(2.83)$ & $\mathrm{Z}=-3.08$ & $p=0.002$ \\
\hline PSWQ [Mean (SD)] & $57.75(13.59)$ & $47.08(15.25)$ & $\mathrm{Z}=2.59$ & $p=0.01$ \\
\hline MMSE [Mean (SD)] & $29.08(0.99)$ & $29.08(0.99)$ & NS & \\
\hline CIRS-G [Mean (SD)] & $5.08(3.12)$ & $5.08(3.12)$ & NS & \\
\hline RSQ[Mean (SD)] & $41.33(12.79)$ & $38(11)$ & $\mathrm{Z}=-1.49$ & $\mathrm{p}=0.14$ \\
\hline History of MDD & 4 & 4 & NS & \\
\hline RBANS [Mean (SD)] & $105(13.97)$ & $109.42(13.87)$ & $\mathrm{Z}=1.099$ & $\mathrm{p}=0.272$ \\
\hline Duration of illness [Mean (SD)] & $16.33(20.47)$ & $16.33(20.47)$ & NS & \\
\hline
\end{tabular}

Table 1. Participant Demographics and Clinical data. Note: NS - Non-significant, HAMA: Hamilton Anxiety Rating Scale, HAM-D: Hamilton Depression Rating Scale, PSWQ: Penn State Worry Questionnaire, MMSE: Mini Mental Examination Scale, CIRS-G: Cumulative Illness Rating Scale for Geriatrics, RSQ: Response Style Questionnaire, RBANS: Repeatable Battery for the Assessment of Neuropsychological Status. $Z=$ Wilcoxon $\mathrm{Z}$ statistics

Normalized cortical thickness in the six ROIs did not differ between pre- and post-treatment: right pars triangularis $(\mathrm{Z}=-0.78, \mathrm{p}=0.43)$, right pars opercularis $(\mathrm{Z}=-0.31$, $\mathrm{p}=0.75)$, left dorsal ACC ( $\mathrm{Z}=-0.55, \mathrm{p}=0.58)$, left rostral ACC $(\mathrm{Z}=-0.63$, $\mathrm{p}=0.53)$, left medial orbitofrontal $(\mathrm{Z}=-0.16, \mathrm{p}=0.88)$, and right medial orbitofrontal $(\mathrm{Z}=0.16, \mathrm{p}=0.88)$. 
Greater pre-treatment normalized cortical thickness in the left dorsal ACC was significantly correlated with improvement in PSWQ pre- to post-treatment $(r(12)=0.765$, $p=0.024$ FDR corrected, see figure 1). This association remained significant even when controlled for presence of comorbid depressive disorder $(r(9)=0.754, p=0.007)$, duration of illness $(r(12)=0.863, p=0.001)$, differences in HAMA $(r(9)=0.75, p=0.008)$, HAMD $(r(9)=0.77, p=0.006)$, and $\operatorname{RSQ}(r(9)=0.76, p=0.006))$. Normalized cortical thickness in left dorsal ACC was not correlated with changes in other clinical variables. Pre-treatment cortical thickness of other ROIs did not correlate significantly with pre- to post-treatment changes in clinical variables.

\begin{tabular}{|l|c|c|c|c|}
\hline Demographics and Clinical data & $\begin{array}{c}\text { Mean (SD) } \\
\mathbf{N = 1 2}\end{array}$ & $\begin{array}{c}\text { Mean (SD) } \\
\mathbf{N}=\mathbf{8}\end{array}$ & $\mathbf{t}(\mathbf{d f})$ & p-value \\
\hline Age [Mean (SD)] & $66.33(5.61)$ & $69(6.67)$ & $\mathrm{t}(18)=-0.96$ & $p=0.35$ \\
\hline Gender (F) & 10 & 3 & - & - \\
\hline Race & $12(\mathrm{~W})$ & $7(\mathrm{~W}), 1(\mathrm{~B})$ & - & - \\
\hline Education [Mean (SD)] & $14.75(2.59)$ & $16.88(2.80)$ & $\mathrm{t}(18)=-1.74$ & $p=0.099$ \\
\hline HAM-A [Mean (SD)] & $21.42(4.42)$ & $20.50(4.11)$ & $\mathrm{t}(18)=0.47$ & $p=0.67$ \\
\hline HAM-D [Mean (SD)] & $12.75(3.59)$ & $12.88(2.64)$ & $\mathrm{t}(18)=-0.084$ & $p=0.93$ \\
\hline PSWQ [Mean (SD)] & $57.75(13.59)$ & $58.25(10.33)$ & $\mathrm{t}(18)=-.088$ & $p=0.93$ \\
\hline CIRS-G [Mean (SD)] & $5.08(3.12)$ & $6.38(1.59)$ & $\mathrm{t}(18)=-1.08$ & $p=0.297$ \\
\hline RSQ [Mean (SD)] & $39.50(13.62)$ & $36.63(10.41)$ & $\mathrm{t}(18)=0.51$ & $p=0.62$ \\
\hline MMSE [Mean (SD)] & $29.08(0.99)$ & $28.63(1.06)$ & $\mathrm{t}(18)=0.98$ & $p=0.34$ \\
\hline RBANS [Mean (SD)] & $105(13.97)$ & $101.38(13.15)$ & $\mathrm{t}(18)=0.58$ & $p=0.57$ \\
\hline History of MDD & $16.33(20.47)$ & $29.63(23.41)$ & $\mathrm{t}(18)=-1.34$ & $p=0.11$ \\
\hline Duration of illness [Mean (SD)] & 4 & & & - \\
\hline
\end{tabular}

Table 2. Comparison of demographic and clinical data between our sample of $\mathrm{N}=12$ and the rest of the GAD from the entire study sample $(\mathrm{N}=8)$ 


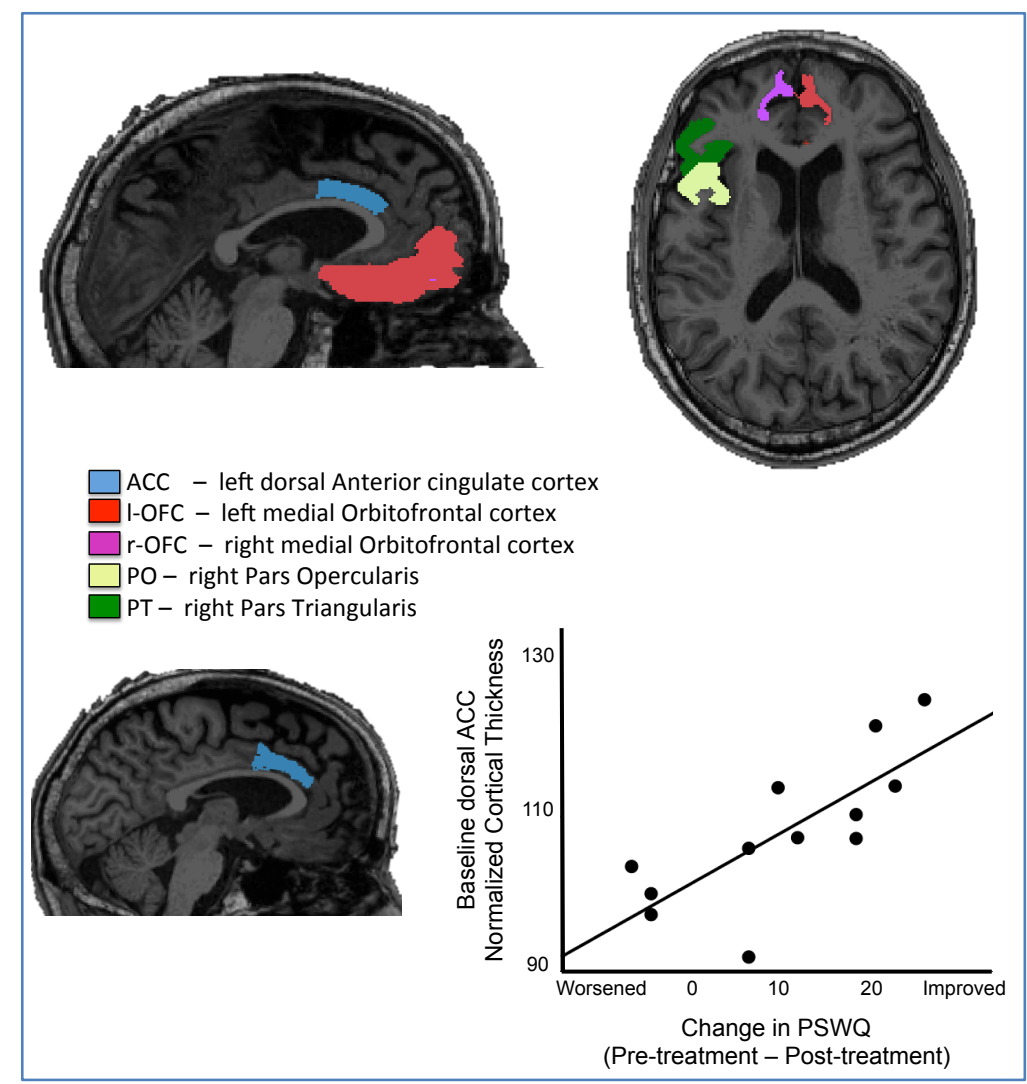

Figure 1. Regions of interest explored in this study (Upper panel). Lower panel: dorsal anterior cingulate cortex ROI (left) and correlation between baseline normalized cortical thickness in left dorsal ACC and change in PSWQ (right). The mean cortical thickness before normalization was 108.16 (SD 9.60) $\mathrm{mm}$.

\section{Discussion}

In this study, worry improvement post-treatment was positively associated with greater pre-treatment dACC cortical thickness. Notably, several clinical markers improved over the twelve weeks of treatment (e.g., global anxiety, worry severity, depression severity), while trait characteristics such as rumination remained unchanged.

The ACC plays a vital role in a number of clinical disorders $[6,19,35,38]$. It is associated with emotional regulation, conflict monitoring [16], error processing and prevention [26], and often the responses to errors and conflicts are affected by emotion. Deficits in emotion regulation in multiple mood and anxiety disorders have often been associated with structural and functional changes in the dorsal ACC [3, 21, 42]. Functional MRI studies have shown lower dorsal anterior cingulate cortical activity during emotional regulation in GAD participants (Blair, 2012) and generating 
fear/anxiety responses (Etkin, 2011). The association between improvement in worry and dACC cortical thickness may be interpreted in the context of the emotion dysregulation theory of GAD [30]. With reduced dACC activity and cortical thickness, there is greater difficulty inhibiting negative stimuli due to chronic worry which is associated with emotional dysregulation. Chronic hyperarousal in the emotional regulation circuitry might diminish attentional resources and emotional response which is associated with lower ACC activation [35].

Recent studies have also emphasized the role of ACC in modulating autonomic and cognitive responses in association with worry [28]. A previous study reported greater cerebral blood flow $(\mathrm{CBF})$ in $\mathrm{dACC}$ during suppression of worry in older non-anxious adults when compared with older GAD [1]. In healthy individuals, cognitive reappraisal has been positively correlated with ACC volumes [21]. While in older adults, preserving the cortical thickness of ACC has been associated with the emotion preservation of aging [29]. This observation was also seen with ACC volume as well. When compared to healthy controls, the volume of the dACC was significantly lower in individuals with clinical disorders [3] [21, 40]. Whether the causal relationship between decreased dACC volume and psychopathology is unidirectional or bi-directional is unclear; in this study, lower dACC was associated with greater levels of worry and lower response to pharmacotherapy.

This study has several limitations including: a small sample size, the open label design, a predominantly Caucasian and female sample, the lack of categorical assessments following treatment, and limited information regarding lifestyle changes and medication compliance. While we have used a previous diagnostic criteria (DSM IV), due to the overlap in symptom criteria in DSM V we do not believe this to be a strong limitation, however it may impact these findings. It is not clear if the non-significant result associated with other ROIs is due to the sample size, an aging effect or the unique role of the dACC. Future studies exploring the optimum duration of pharmacotherapy in late-life GAD or the association of $\mathrm{dACC}$ structural and functional neuroanatomy with post-treatment efficacy of emotion regulation strategies may help shed light on the role of $\mathrm{dACC}$ in the treatment of severe worry and late-life GAD. 


\section{Conclusions}

These results suggest that the $\mathrm{AACC}$ is pivotal for worry regulation and its structural and functional neuroanatomy may be further investigated as a potential biomarker for treatment response in late-life GAD.

Acknowledgments: Brain and Behavior (NARSAD) Young Investigator Award, NIMH K23 086686, R01 MH076079, NIMH R01 MH108509.

Financial disclosures: Anusha Rangarajan, Helmet Karim, Sarah Walker and Carmen Andreescu do not have any potential conflicts of interest to acknowledge. Howard Aizenstein has received research support from Novartis Pharmaceuticals

\section{Author Contributions}

(a) Conception and design: Carmen Andreescu, Howard Aizenstein

(b) Acquisition of data: Carmen Andreescu, Sarah Walker

(c) Analysis and interpretation of data: Anusha Rangarajan, Helmet Karim, Dana Tudorascu, Carmen Andreescu

(e) Revising for intellectual content: Anusha Rangarajan, Helmet Karim, Dana Tudorascu, Carmen Andreescu, Howard Aizenstein

(f) Final approval of the completed manuscript: Anusha Rangarajan, Helmet Karim, Sarah Walker, Dana Tudorscu, Carmen Andreescu, Howard Aizenstein 


\section{References}

[1] C. Andreescu, J.J. Gross, E. Lenze, K.D. Edelman, S. Snyder, C. Tanase, H. Aizenstein, Altered cerebral blood flow patterns associated with pathologic worry in the elderly, Depress Anxiety 28 (2011) 202-209.

[2] C. Andreescu, D. Tudorascu, L.K. Sheu, A. Rangarajan, M.A. Butters, S. Walker, R. Berta, T. Desmidt, H. Aizenstein, Brain structural changes in late-life generalized anxiety disorder, Psychiatry Res 268 (2017) 15-21.

[3] T. Asami, F. Hayano, M. Nakamura, H. Yamasue, K. Uehara, T. Otsuka, T. Roppongi, N. Nihashi, T. Inoue, Y. Hirayasu, Anterior cingulate cortex volume reduction in patients with panic disorder, Psychiatry Clin Neurosci 62 (2008) 322330.

[4] S.A. Beaudreau, R. O'Hara, Late-life anxiety and cognitive impairment: a review, Am J Geriatr Psychiatry 16 (2008) 790-803.

[5] Y. Benjamini, Y. Hochberg, Controlling the false discovery rate: a practical and powerful approach to multiple testing, Journal of the royal statistical society. Series B (Methodological) (1995) 289-300.

[6] K.S. Blair, M. Geraci, B.W. Smith, N. Hollon, J. DeVido, M. Otero, J.R. Blair, D.S. Pine, Reduced dorsal anterior cingulate cortical activity during emotional regulation and top-down attentional control in generalized social phobia, generalized anxiety disorder, and comorbid generalized social phobia/generalized anxiety disorder, Biol Psychiatry 72 (2012) 476-482.

[7] D. Blazer, George, L. K., \& Hughes, D. , The epidemiology of anxiety disorders: an age comparison., Anxiety in the Elderly: Treatment and Research (1991) 17-30.

[8] M.A. Butters, R.K. Bhalla, C. Andreescu, J.L. Wetherell, R. Mantella, A.E. Begley, E.J. Lenze, Changes in neuropsychological functioning following treatment for late-life generalised anxiety disorder, Br J Psychiatry 199 (2011) 211-218.

[9] A. Canuto, K. Weber, M. Baertschi, S. Andreas, J. Volkert, M.C. Dehoust, S. Sehner, A. Suling, K. Wegscheider, B. Ausin, M.J. Crawford, C. Da Ronch, L. Grassi, Y. Hershkovitz, M. Munoz, A. Quirk, O. Rotenstein, A.B. Santos-Olmo, A. Shalev, J. Strehle, H.U. Wittchen, H. Schulz, M. Harter, Anxiety Disorders in Old Age: Psychiatric Comorbidities, Quality of Life, and Prevalence According to Age, Gender, and Country, Am J Geriatr Psychiatry 26 (2018) 174-185.

[10] J.M. Cisler, E.H. Koster, Mechanisms of attentional biases towards threat in anxiety disorders: An integrative review, Clinical psychology review 30 (2010) 203-216. 
[11] D.L. Collins, P. Neelin, T.M. Peters, A.C. Evans, Automatic 3D intersubject registration of MR volumetric data in standardized Talairach space, J Comput Assist Tomogr 18 (1994) 192-205.

[12] P.T. Costa, Jr., R.R. McCrae, Age differences in personality structure: a cluster analytic approach, J Gerontol 31 (1976) 564-570.

[13] A.M. Dale, B. Fischl, M.I. Sereno, Cortical surface-based analysis. I. Segmentation and surface reconstruction, Neuroimage 9 (1999) 179-194.

[14] W.C. Drevets, J.L. Price, M.L. Furey, Brain structural and functional abnormalities in mood disorders: implications for neurocircuitry models of depression, Brain Struct Funct 213 (2008) 93-118.

[15] K. Duff, J.D. Humphreys Clark, S.E. O'Bryant, J.W. Mold, R.B. Schiffer, P.B. Sutker, Utility of the RBANS in detecting cognitive impairment associated with Alzheimer's disease: sensitivity, specificity, and positive and negative predictive powers, Arch Clin Neuropsychol 23 (2008) 603-612.

[16] A. Etkin, T. Egner, R. Kalisch, Emotional processing in anterior cingulate and medial prefrontal cortex, Trends Cogn Sci 15 (2011) 85-93.

[17] M.B. First, R.L. Spitzer, M. Gibbon, J.B. Williams, M. Davies, J. Borus, M.J. Howes, J. Kane, H.G. Pope Jr, B. Rounsaville, The structured clinical interview for DSM-III-R personality disorders (SCID-II). Part II: Multi-site test-retest reliability study, Journal of personality disorders 9 (1995) 92-104.

[18] B. Fischl, M.I. Sereno, A.M. Dale, Cortical surface-based analysis. II: Inflation, flattening, and a surface-based coordinate system, Neuroimage 9 (1999) 195-207.

[19] K.D. Fitzgerald, R.C. Welsh, W.J. Gehring, J.L. Abelson, J.A. Himle, I. Liberzon, S.F. Taylor, Error-related hyperactivity of the anterior cingulate cortex in obsessive-compulsive disorder, Biol Psychiatry 57 (2005) 287-294.

[20] F.S. Folstein MF, McHugh PR Mini-Mental State: a practical method for grading the cognitive state of patients for the clinician, J Psychiatr Res 12 (1975) 189-198.

[21] N.R. Giuliani, E.M. Drabant, J.J. Gross, Anterior cingulate cortex volume and emotion regulation: is bigger better?, Biol Psychol 86 (2011) 379-382.

[22] M. Hamilton, The assessment of anxiety states by rating, Br J Med Psychol 32 (1959) 50-55.

[23] M. Hamilton, A rating scale for depression, J Neurol Neurosurg Psychiatry 23 (1960) 56-62.

[24] E.A. Hoge, A. Ivkovic, G.L. Fricchione, Generalized anxiety disorder: diagnosis and treatment, BMJ 345 (2012) e7500. 
[25] R.Y. Hong, Worry and rumination: differential associations with anxious and depressive symptoms and coping behavior, Behav Res Ther 45 (2007) 277-290.

[26] N. Ichikawa, G.J. Siegle, N.P. Jones, K. Kamishima, W.K. Thompson, J.J. Gross, H. Ohira, Feeling bad about screwing up: emotion regulation and action monitoring in the anterior cingulate cortex, Cogn Affect Behav Neurosci 11 (2011) 354-371.

[27] H. Le Roux, M. Gatz, J.L. Wetherell, Age at onset of generalized anxiety disorder in older adults, Am J Geriatr Psychiatry 13 (2005) 23-30.

[28] E. Makovac, F. Meeten, D.R. Watson, A. Herman, S.N. Garfinkel, D.C. H, C. Ottaviani, Alterations in Amygdala-Prefrontal Functional Connectivity Account for Excessive Worry and Autonomic Dysregulation in Generalized Anxiety Disorder, Biol Psychiatry 80 (2016) 786-795.

[29] M. Mather, The emotion paradox in the aging brain, Ann N Y Acad Sci 1251 (2012) 33-49.

[30] D.S. Mennin, R.M. Holaway, D.M. Fresco, M.T. Moore, R.G. Heimberg, Delineating components of emotion and its dysregulation in anxiety and mood psychopathology, Behav Ther 38 (2007) 284-302.

[31] T.J. Meyer, M.L. Miller, R.L. Metzger, T.D. Borkovec, Development and validation of the Penn State Worry Questionnaire, Behav Res Ther 28 (1990) 487495 .

[32] M.D. Miller, C.F. Paradis, P.R. Houck, S. Mazumdar, J.A. Stack, A.H. Rifai, B. Mulsant, C.F. Reynolds, 3rd, Rating chronic medical illness burden in geropsychiatric practice and research: application of the Cumulative Illness Rating Scale, Psychiatry Res 41 (1992) 237-248.

[33] J. Mohlman, R.B. Price, D.A. Eldreth, D. Chazin, D.M. Glover, W.R. Kates, The relation of worry to prefrontal cortex volume in older adults with and without generalized anxiety disorder, Psychiatry Res 173 (2009) 121-127.

[34] S. Nolen-Hoeksema, J. Morrow, B.L. Fredrickson, Response styles and the duration of episodes of depressed mood, J Abnorm Psychol 102 (1993) 20-28.

[35] S.S. Pillay, S.A. Gruber, J. Rogowska, N. Simpson, D.A. Yurgelun-Todd, fMRI of fearful facial affect recognition in panic disorder: the cingulate gyrus-amygdala connection, J Affect Disord 94 (2006) 173-181.

[36] S.C. Segerstrom, A.R. Roach, D.R. Evans, L.J. Schipper, A.K. Darville, The structure and health correlates of trait repetitive thought in older adults, Psychology and aging 25 (2010) 505-515.

[37] L. Staner, Sleep and anxiety disorders, Dialogues Clin Neurosci 5 (2003) 249-258. 
[38] W.T. To, D. De Ridder, T. Menovsky, J. Hart, S. Vanneste, The role of the dorsal Anterior Cingulate Cortex (dACC) in a cognitive and emotional counting Stroop task: Two cases, Restor Neurol Neurosci 35 (2017) 333-345.

[39] D.P. Tromp, D.W. Grupe, D.J. Oathes, D.R. McFarlin, P.J. Hernandez, T.R. Kral, J.E. Lee, M. Adams, A.L. Alexander, J.B. Nitschke, Reduced structural connectivity of a major frontolimbic pathway in generalized anxiety disorder, Arch Gen Psychiatry 69 (2012) 925-934.

[40] N. Vasic, H. Walter, A. Hose, R.C. Wolf, Gray matter reduction associated with psychopathology and cognitive dysfunction in unipolar depression: a voxel-based morphometry study, J Affect Disord 109 (2008) 107-116.

[41] J.L. Wetherell, S.R. Thorp, T.L. Patterson, S. Golshan, D.V. Jeste, M. Gatz, Quality of life in geriatric generalized anxiety disorder: a preliminary investigation, $\mathrm{J}$ Psychiatr Res 38 (2004) 305-312.

[42] K. Yucel, M.C. McKinnon, R. Chahal, V.H. Taylor, K. Macdonald, R. Joffe, G.M. MacQueen, Anterior cingulate volumes in never-treated patients with major depressive disorder, Neuropsychopharmacology 33 (2008) 3157-3163.

[43] Y. Zhang, C. Wang, C. Sun, X. Zhang, Y. Wang, H. Qi, F. He, X. Zhao, B. Wan, J. Du, D. Ming, Neural complexity in patients with poststroke depression: A resting EEG study, J Affect Disord 188 (2015) 310-318. 